Gynäkologe 2009 • 42:5-6

DOI 10.1007/s00129-008-2273-y

Online publiziert: 18. Dezember 2008

(c) Springer Medizin Verlag 2008

K. Diedrich ${ }^{1} \cdot$ P. Husslein ${ }^{2}$ ' W. Jonat ${ }^{3} \cdot$ M.K. Bohlmann ${ }^{1}$

${ }^{1}$ Klinik für Frauenheilkunde und Geburtshilfe, Universitätsklinikum Schleswig-Holstein - Campus Lübeck

${ }^{2}$ Abteilung für Geburtshilfe und feto-maternale Medizin, Universitäts-Klinik für Frauenheilkunde, Wien

${ }^{3}$ Universitätsfrauenklinik Kiel

\title{
Habituelle Aborte
}

\section{Aktuelle diagnostische und therapeutische Aspekte eines komplexen Krankheitsbildes}

tumsfaktoren oder TNFa-Inhibitoren zu finden, für deren Anwendung oder gar Wirksamkeit bei habituellen Aborten in nationalen wie internationalen Fachzeitschriften keinerlei Erfahrungsberichte existieren und die, wie im Fall der TNFaBlocker, sogar im Verdacht stehen, kanzerogen zu wirken. Gerade bei den uns anvertrauten Schwangeren mit all den potenziellen Langzeitfolgen gilt es, das Prinzip des „Primum nihil nocere“ $z$ u beachten.

Da insgesamt „nur“ weniger als $2 \%$ aller Partnerschaften von wiederholten Fehlgeburten betroffen sind, kann aufgrund der multifaktoriellen Genese der Abortneigung die Vorstellung der Paare in interdisziplinären Sprechstunden ein Schlüssel zum erfolgreichen Ausgang einer Folgegravidität sein. So besteht in vielen Fällen durch eine systematische Abklärung und Einleitung einer evidenzbasierten Therapie vor und während einer Folgegravidität eine realistische Chance auf die Erfüllung des Kinderwunsches. Ein konsequentes Vorgehen ist dabei umso wichtiger, da bei bestimmten Krankheitsbildern - z. B. dem Antiphospholipid-Syndrom - unbehandelt ein sehr hohes Fehlund Totgeburtsrisiko besteht, bei einer gezielten Therapie in einer Folgeschwangerschaft hingegen mit einer hohen Austragungsrate zu rechnen ist.

Im Rahmen dieses Themenheftes „Habituelle Aborte“ werden diagnostische Vorgehensweisen sowie aktuelle Therapiestandards und Handlungsempfehlungen bei wiederholten Fehlgeburten dargestellt und bis dato nicht etablierte Ansätze einer kritischen Diskussion unterzogen.

\section{○ Die Abklärung parentaler Chromosomenanomalien ist ein wichtiger Bestandteil der Diagnostik}

Bei Patientinnen mit wiederholten Fehlgeburten sind chromosomale embryonale Abberationen seltener als bei sporadisch auftretenden Aborten. Aufgrund ihrer hohen Bedeutung und der fehlenden Therapieoptionen gehört jedoch die Abklärung vererbbarer Chromosomenanomalien der Eltern zu den wichtigsten Bestandteilen des diagnostischen Vorgehens. Solche „Genetische[n] Ursachen“ eines habituellen Abortgeschehens werden von C. Tempfer dargestellt.

Angeborene anatomische Veränderungen, z. B. ein Uterusseptum, werden mit einem sehr hohen Risiko für Schwangerschaftskomplikationen - bis zu 80\% in Verbindung gebracht. A. Hornemann et al. beschreiben in ihrem Beitrag „Anatomische Ursachen habitueller Aborte“ nicht nur den aktuellen Kenntnisstand zur Häufigkeit der verschiedenen uterinen Veränderungen, sondern beleuchten ebenso therapeutische Optionen der jeweiligen Malformationen, durch die in vielen Fällen eine signifikante Verbesserung der Lebendgeburtrate erreicht werden kann.

Die Diagnostik „Thrombophile[r] Gerinnungsstörungen als Risikofaktoren für habituelle Aborte" sowie die sich hiernetforen beispielsweise Berichte Betroffener über die Anwendung von Wachs- 
aus ergebenden medikamentösen Ansätze werden - auch vor dem Hintergrund aktueller Studien zur Abortprävention mit Heparin - von M. Bohlmann et al. dargestellt.

Die komplexen Zusammenhänge zwischen „Schwangerschaft und maternalem Immunsystem“" werden von der Arbeitsgruppe um B. Toth und C. Scholz ausführlich erläutert, wobei ein Schwerpunkt der Diskussion auf der klinischen Relevanz verschiedener immunmodulatorischer Therapieansätze liegt.

Ein oftmals unterschätztes Krankheitsbild sind die zum Teil komplexen „Endokrinopathien als Ursachen habitueller Aborte", deren diagnostische und therapeutische Optionen von R. Speer und J.W. Dudenhausen umfassend wiedergegeben und kommentiert werden.

Nicht zu unterschätzen sind interdisziplinär anzugehende „Psychische Faktoren bei habituellen Aborten“, mit denen sich C. Schulz-Du Bois und A. SchulzDu Bois in ihrem Beitrag ausführlich beschäftigen.

Abschließend weisen A. Witt und H. Kiss in ihrem Beitrag „Infektionen in Gynäkologie und Geburtshilfe“ auf $\mathrm{Zu}$ sammenhänge zwischen genitalen Entzündungen und Schwangerschaftskomplikationen sowie auf Therapiestrategien während der Gravidität hin.

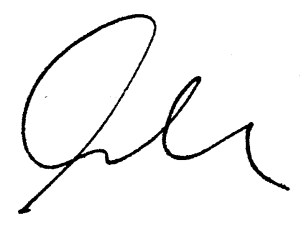

Prof. Dr. K. Diedrich

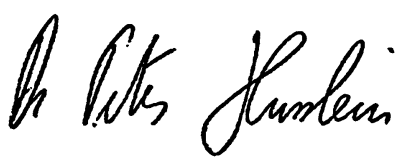

Prof. Dr. P. Husslein

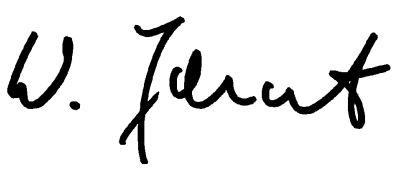

Prof. Dr. W. Jonat

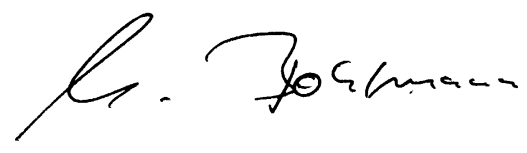

Dr. M.K. Bohlmann

\section{Korrespondenzadresse}

\section{Dr. M.K. Bohlmann}

Klinik für Frauenheilkunde und Geburtshilfe,

Universitätsklinikum Schleswig-

Holstein - Campus Lübeck

Ratzeburger Allee 160, 23538 Lübeck

michael.bohlmann@uk-sh.de

\section{Exklusiv für Abonnenten :}

\section{Nutzen Sie das Online-Archiv von Der Gynäkologe}

Ihre Vorteile:

- Komfortable und schnelle Recherche nach Themen, Autoren, Suchbegriffen

— Ob unterwegs oder am eigenen PC: Zugriff überall und jederzeit

- Online First: Lesen Sie die aktuellsten Beiträge schon vor Erscheinen des gedruckten Heftes online

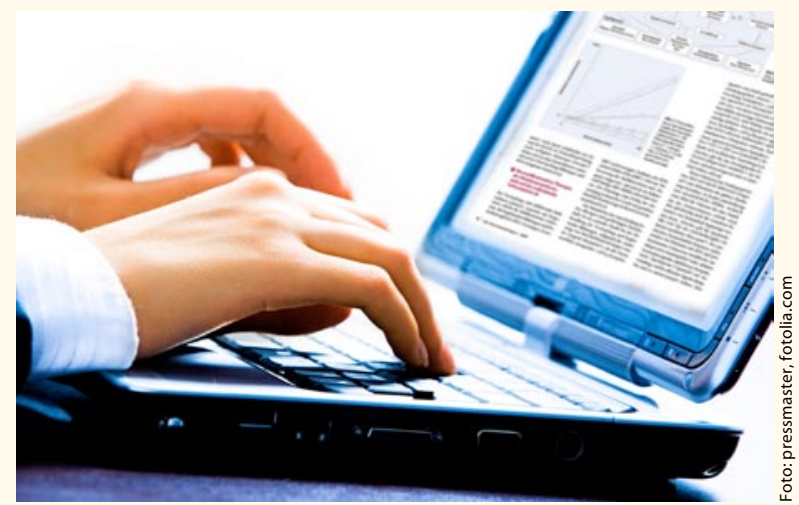

